\title{
Early Outcomes of Transection of Secondary Chordae with Downsized Ring Annuloplasty in Low Recurrence Risk Severe Ischemic Mitral Regurgitation Hussein Derbala, Alaa Omar, Ayman Gado, Hesham Zayed Saleh* \\ Department Cardiothoracic Surgery, Kasr Al Ainy, Faculty of Medicine, Cairo University, Egypt. \\ *Corresponding Author: Hesham Zayed Saleh, Email: hzayed@ hotmail.com
}

\begin{abstract}
Background: Relying solely on restrictive annuloplasty for the repair of ischemic mitral regurgitation is associated with an unacceptable rate of recurrence. Transection of secondary chordae as an adjunctive procedure has been proposed to improve results.

Patient and methods: This is a prospective observational study including 20 patients who underwent mitral repair using downsized annuloplasty ring and transection of the secondary chordae, at the time of CABG at the Cardiothoracic Surgery Department of Cairo University Hospitals through the period between March 2016 and October 2018. The primary outcome being examined was the recurrence of significant mitral regurgitation at 6 months postoperatively. Secondary outcomes included coaptation length and ejection fraction.

Results: The mean age was $57.5 \pm 6.9$ years. The mean ejection fraction was $45.1 \pm 11 \%$. There was one early mortality $(5 \%)$. At 6 months postoperatively, there was no incidence of recurrence of significant mitral regurgitation. Coaptation length was quite satisfactory $(7.2 \pm 0.67 \mathrm{~mm})$.

Conclusion: Transection of secondary chordae is a safe and simple option. It may be beneficial when performed in combination with downsized ring annuloplasty in patients with low-risk ischemic mitral regurgitation.
\end{abstract}

Keywords: Ischemic mitral regurgitation, Mitral repair, Secondary chordae.

\section{INTRODUCTION}

Mitral regurgitation is a common complication of myocardial infarction ${ }^{(\mathbf{1})}$. Independent of age, gender or ejection fraction, significant ischemic mitral regurgitation (IMR) is associated with an increased risk of heart failure and death in the years following the initial ischemic event ${ }^{(2)}$.

Given the heterogeneity of the underlying mechanisms precipitating chronic IMR in different subgroups of patients, the ideal surgical management remains debatable ${ }^{(3,4)}$.

Undersized ring annuloplasty as described by Bolling et al. ${ }^{(5)}$, at the time of coronary bypass surgery, was the commonest used surgical procedure. However, the results of the Cardiothoracic Surgical Trials Network (CTSN) trial raised serious concerns about the rate of recurrence of mitral regurgitation following such a procedure. In this randomized trial, the recurrence rate of significant mitral regurgitation reached $58.8 \%$ at two years, and was associated with a significantly higher incidence of heart failure and hospital readmissions, when compared to the group of patients who underwent mitral valve replacement ${ }^{(6)}$.

Such high rates of recurrence led to increasing interest in previously published reports pointing out preoperative echocardiographic parameters, to predict the risk of recurrence of mitral regurgitation after mitral repair in these patients. These echocardiographic parameters included among others, the tenting height, tenting area and interpapillary distance, which proved reliable and easy to measure ${ }^{(7-10)}$.

There was also an increasing adoption of more complex forms of mitral repair, to improve long-term outcomes ${ }^{(10-12)}$.
Among these, resection of secondary chordae proved to be a simple and effective option ${ }^{(12,13)}$.

In the current study we described the early outcomes of transection of secondary chordae combined with downsized ring annuloplasty, in patients with low recurrence risk IMR undergoing coronary artery bypass grafting (CABG).

\section{PATIENTS AND METHODS}

This is a prospective observational study including 20 patients who underwent mitral repair using downsized annuloplasty ring and transection of the secondary chordae, at the time of CABG at the Cardiothoracic Surgery Department of Cairo University Hospitals in the period between March 2016 and October 2018.

During this period, a total of 26 consecutive patients underwent this procedure. 6 patients were excluded.

Exclusion criteria: being considered high risk for recurrence of significant mitral regurgitation based on preoperative echocardiographic assessment, previous cardiac surgery, primary mitral valve lesions of rheumatic or degenerative origin identified on preoperative echocardiography, patients with other valvular lesions and patients with a left ventricular ejection fraction less than $25 \%$.

The remaining 20 patients were included in the study and their individual files were reviewed to extract clinical, operative and outcome data. 


\section{Ethical Consideration:}

The study was approved by the Local Ethical Committee of Cairo University. Written consent was obtained from every patient prior to the procedures. This study has been carried out in accordance with the code of Ethics of the World Medical Association (Declaration of Helsinki) for studies involving humans.

\section{Outcomes of interest:}

Preoperative, operative and postoperative characteristics were collected. The primary outcome was the presence of significant residual mitral regurgitation at six months follow-up. Secondary outcomes included mitral valve coaptation length and ejection fraction.

\section{Echocardiographic Assessment:}

Transthoracic echocardiography was performed in all patients preoperatively, within a week postoperatively and at follow-up 6 months after the surgery. Comprehensive assessment included measurement of left ventricular and left atrial dimensions and ejection fraction. The severity of mitral regurgitation was graded in a semiquantitative fashion as grade (0), grade (1) mild, grade (2) moderate, grade (3) moderate to severe, and grade (4) severe. Severe mitral regurgitation was defined as mitral regurgitation with a vena contracta width $\geq 70 \mathrm{~mm}$.

Geometric mitral valve parameters used to categorize patients into low-risk versus high risk for recurrence included the following: (1) Mitral valve tenting area defined as the triangular area bound by the annular plane and the valve leaflets in the transthoracic parasternal long-axis view in systole. This was considered high-risk if $\geq 2.5 \mathrm{~cm}^{2}$ (7). (2) The tenting height defined as the distance from the annular plane to the coaptation point in the transthoracic parasternal long-axis view in systole. This was considered high-risk if $\geq 1 \mathrm{~cm}$. 3. The interpapillary muscle distance was defined as the distance between the anterolateral and the posteromedial heads as measured in the parasternal short-axis view in diastole. This was considered highrisk if $\geq 20 \mathrm{~mm}^{(8)}$.

Coaptation height was measured in postoperative echoes in the parasternal long-axis view and was defined as the length of the coaptation zone between both leaflets at the level of the middle scallop.

\section{Operative technique:}

All patients were operated through median sternotomy with aorto-bicaval cannulation. Myocardial protection was ensured by intermittent cold blood cardioplegia and moderate systemic hypothermia. The mitral valve was approached through left atriotomy. All patients received coronary bypass grafts. Mitral repair was started by placing the interrupted 2-0 braided sutures alongside the circumference of the mitral annulus, to improve the exposure of the mitral valve apparatus. This was followed by identification of strut secondary chordae to the belly of the anterior leaflet, P3 scallop of the posterior leaflet and posterior commissure. Identified secondary chordae were carefully separated from the primary chordae using a nerve hook before resecting them. Saline test was then performed and the mitral valve inspected for any indentation on the atrial side of the leaflets denoting any missed secondary chords causing tethering and requiring resection. Mitral repair was then completed by the insertion of a rigid complete annuloplasty ring: Carpentier-Edwards Physio ring (Edwards Life sciences, Irving, CA, USA), which was two sizes below the size determined by the intraoperative sizer.

\section{Statistical Analysis}

Data were analyzed using the statistical package for social sciences, version 20.0 (SPSS Inc., Chicago, Illinois, USA). Continuous variables were expressed as mean \pm standard deviation (SD). Categorical variables were expressed as numbers and percentages. Comparison of quantitative variables was done using student $t$ test while comparison of categorical data was done using Chi-square test $\left(\mathrm{X}^{2}\right)$. A difference was considered significant when $\mathrm{p} \leq 0.05$.

\section{RESULTS}

Table (1) outlined the preoperative characteristics of patients with a mean age of $57.5 \pm 6.9$ years and a mean ejection fraction of $45.1 \pm 11 \%$. Table (2) outlines some of the operative data. Most of the patients (55\%) received three coronary bypass grafts in addition to mitral repair.

Table (3) outlined the postoperative outcomes, with one fatality $(5 \%)$. The fatality case followed a low cardiac output state complicating a postoperative myocardial infarction. Table (4) outlined the echocardiographic follow-up data at 6 months. The follow up was completed in 18 patients $(94.7 \%$ of the surviving patients). None of the patients developed significant mitral regurgitation. There was a noticeable improvement in ejection fraction in patients. The positive finding was a coaptation length that was quite satisfactory $(7.2 \pm 0.6)$.

Table (1): Preoperative patients' characteristics

\begin{tabular}{|l|c|}
\hline & $\begin{array}{c}\text { Preoperative } \\
\text { Variables }\end{array}$ \\
\hline Age (years) & $57.5 \pm 6.9$ \\
\hline Sex (Females) & $10(50 \%)$ \\
\hline Diabetes Mellitus & $14(70 \%)$ \\
\hline Hypertension & $15(75 \%)$ \\
\hline Recent Myocardial Infarct & $6(30 \%)$ \\
\hline NYHA: & \\
Class II & $8(40 \%)$ \\
Class III & $12(60 \%)$ \\
\hline LVEDD & $6.1 \pm 0.86$ \\
\hline LVESD & $4.4 \pm 1$ \\
\hline EF $(\%)$ & $45.1 \pm 11$ \\
\hline
\end{tabular}


Table (2): Operative data

\begin{tabular}{|l|c|}
\hline & $\mathbf{N}(\mathbf{\%})$ \\
\hline Number of grafts: & $2(10 \%)$ \\
Single graft & $5(25 \%)$ \\
Two grafts & $11(55 \%)$ \\
Three grafts & $2(10 \%)$ \\
Four grafts & $113.5 \pm 23.3$ \\
\hline Cross clamp time & $147 \pm 28.4$ \\
\hline Total bypass time & \\
\hline
\end{tabular}

Table (3): Postoperative outcomes

\begin{tabular}{|l|c|}
\hline & $\mathbf{N}(\mathbf{\%})$ \\
\hline Mortality & $1(5 \%)$ \\
\hline Stroke & $0(0 \%)$ \\
\hline Myocardial infarction & $1(5 \%)$ \\
\hline RF requiring dialysis & $1(5 \%)$ \\
\hline Deep wound infection & $1(5 \%)$ \\
\hline Atrial fibrillation & $5(25 \%)$ \\
\hline
\end{tabular}

Table (4): Echocardiographic follow-up data at 6 months

\begin{tabular}{|l|c|}
\hline & Mean \pm SD \\
\hline MR +3 or more & $0(0 \%)$ \\
\hline Coaptation length $(\mathrm{mm})$ & $7.2 \pm 0.6$ \\
\hline Ejection fraction $(\%)$ & $49 \pm 6.4$ \\
\hline
\end{tabular}

\section{DISCUSSION}

The main mechanisms underlying the development of chronic IMR are related to the altered geometry of the mitral valve, which results from left ventricular remodeling following myocardial infarction. Papillary muscle displacement resulting from left ventricular remodeling causes tethering of the mitral valve leaflets and restricted closure resulting in mitral regurgitation ${ }^{(14-16)}$. This tethering of the leaflets is effected mainly through secondary (basal) chordae, which attach to the belly of the anterior mitral leaflet resulting in a characteristic bend, often referred to as 'seagull sign' (15). However this characteristic bend seems to vary between individuals depending on the insertion site of secondary chordae, thus affecting the severity of secondary mitral regurgitation. As demonstrated by Song $\boldsymbol{e t}$ al. ${ }^{(17)}$, the insertion sites of secondary chordae showed significant individual variation, with individuals whose outer secondary chordae attached further to the basal side of the anterior leaflet being more susceptible to more severe mitral regurgitation. There seems to be an individual inborn susceptibility to significant IMR in these patients. With this relatively recent elaboration on the role of secondary chordae in the pathophysiology of IMR, transection of the secondary chordae would be beneficial, at least in some patients.

The beneficial effect of resecting secondary chordae was demonstrated earlier in an experimental sheep model, where cutting the basal chordae was associated with improved leaflet coaptation and marked reduction of secondary mitral regurgitation ${ }^{(18)}$. In a clinical study, Borger et al. ${ }^{(19)}$ found that chordal cutting to be associated with a reduced risk of recurrence of mitral regurgitation at 2-years follow-up when compared to downsized annuloplasty alone. Calafiore et al. ${ }^{(12)}$ demonstrated similar results although in their study the benefit in terms of lesser recurrence of mitral regurgitation was limited to patients with bending angle $<145^{\circ}$ and coaptation depth less than $10 \mathrm{~mm}$. Although, the current study was not designed to demonstrate such benefits, we demonstrated however, similar to the above-mentioned studies that resection of secondary chordae is a simple surgical technique, not adding to the aortic cross-clamp time or operative risk.

One benefit we demonstrated was a very satisfactory coaptation length, which was previously demonstrated to be associated with a better durability of mitral repair, by many authors ${ }^{(\mathbf{9}, 20)}$. Another important finding in the current study was an improvement in left ventricular ejection fraction at follow-up. This finding, which is in line with previously mentioned reports ${ }^{(12,13}$, ${ }^{19)}$ refute previous claims, that resection of secondary chordae may be associated with impairment in ventricular function (21). The longer-term effects of chordal cutting remain to be demonstrated, although at a mean follow-up around 4 years, Murashita et al. ${ }^{(13)}$ demonstrated that adding chordal transection to restrictive annuloplasty resulted in satisfactory results, except in patients with a higher tenting height. Such patients carry a higher mitral regurgitation recurrence risk and were not included in the current study.

\section{CONCLUSION}

Transection of secondary chordae is a safe and simple option. It may be beneficial when performed in combination with downsized ring annuloplasty in patients with low risk ischemic mitral regurgitation. Further studies recruiting higher numbers of patients, with longer follow-up are needed to validate our findings and further characterize patients' subsets that would benefit the most from this procedure.

\section{Financial support and sponsorship: Nil. Conflict of interest: Nil.}

\section{REFERENCES}

1. Barzilai B, Gessler C, Perez J et al. (1988): Significance of Doppler-detected mitral regurgitation in acute myocardial infarction. Am J Cardiol., 61: 220-23.

2. Bursi F, Enriquez-Sarano M, Nkomo V et al. (2005): Heart Failure and death after myocardial infarction in the community: the emerging role of mitral regurgitation. Circulation, 111: 295-301.

3. Baez-Ferrer N, Izquierdo-Gomez M, Mari-Lopez B et al. (2018): Clinical manifestations, diagnosis and treatment of ischemic mitral regurgitation: a review. $\mathbf{J}$ Thorac Dis., 10 (12): 6969-86.

4. Yamazaki S, Numata S, Yaku H (2019): Surgical intervention for ischemic mitral regurgitation: how can we achieve better outcomes. Surg Today, 50 (6): 540-50.

5. Bolling S, Pagani F, Deeb G et al. (1998): Intermediateterm outcome of mitral reconstruction in 
cardiomyopathy. J Thorac Cardiovasc Surg., 115: 38186.

6. Goldstein D, Moskowitz A, Gelijns A et al. (2016): Two-year outcomes of surgical treatment of severe ischemic mitral regurgitation. N Engl J Med., 374: 34453.

7. Magne J, Pibarot P, Dagenais F et al. (2007): Preoperative posterior leaflet angle accurately predicts outcome after restrictive mitral valve annuloplasty for ischemic mitral regurgitation. Circulation, 115: 782-91.

8. Lee A, Acker M, Kubo S et al. (2009): Mechanisms of recurrent functional mitral regurgitation after mitral valve repair in nonischemic dilated cardiomyopathy: importance of distal anterior leaflet tethering. Circulation, 119: 2606-14.

9. Gelsomino S Lorusso R, Caciolli $\mathrm{S}$ et al. (2008): Insights on left ventricular and valvular mechanisms of recurrent ischemic mitral regurgitation after restrictive annuloplasty and coronary artery bypass grafting. J Thorac Cardiovasc Surg., 136: 507-18.

10. Nappi F, Lusini M, Singh S et al. (2019): Risk of ischemic mitral regurgitation recurrence after combined valvular and subvalvular repair. Ann Thorac Surg., 108: 536-43.

11. De Varennes B, Chaturvedi R, Sidhu S et al. (2009): Initial results of posterior leaflet extension for severe type IIIb ischemic mitral regurgitation. Circulation, 119: 2837-43.

12. Calafiore A, Refaie R, Iaco A et al. (2014): Chordal cutting in ischemic mitral regurgitation: A propensitymatched study. J Thorac Cardiovasc Surg., 148: 41-6.

13. Murashita T, Okada Y, Kanemitsu H et al. (2014): Midterm outcomes of chordal cutting in combination with downsized ring annuloplasty for ischemic mitral regurgitation. Ann Thorac Cardiovasc Surg., 20: 100815.

14. Grigioni F, Enriquez-Sarano M, Zehr K et al. (2001): Ischemic mitral regurgitation. Long-term outcome and prognostic implications with quantitative Doppler assessment. Circulation, 103:1759-64.

15. Levine R, Schwammenthal E (2005): Ischemic mitral regurgitation on the threshold of a solution: from paradoxes to unifying concepts. Circulation, 112: 74558.

16. Hung J (2013): Ischemic (Functional) mitral regurgitation. Cardiol Clin., 31: 231-36.

17. Song J, Kim J, Ha T et al. (2015): Basal chordae sites on the mitral valve determine the severity of secondary mitral regurgitation. Heart, 101: 1024-31.

18. Messas E, Guerrero J, Handschumacher $M$ et al. (2001): Chordal cutting: a new therapeutic approach for ischemic mitral regurgitation. Circulation, 104: 1958-63.

19. Borger M, Murphy $P$ Alam A et al. (2007): Initial results of the chordal-cutting operation for ischemic mitral regurgitation. J Thorac Cardiovasc Surg., 133: 1483-92.

20. Falk V, Seeburger J, Czesla M et al. (2008): How does the use of polytetrafluoroethylene neochordae for posterior mitral valve proplapse (loop technique) compare with leaflet resection? A prospective randomized trial. J Thorac Cardiovasc Surg., 136: 120006.

21. Rodriguez F, Langer F, Harrington $K$ et al. (2004): Importance of mitral valve second-order chordae for left ventricular geometry, wall thickening mechanics, and global systolic function. Circulation, 110 (11): 115 -22. 